\title{
EFFECTS OF VARIABLE TEMPERATURE ON MHD FREE CONVECTIVE FLOW IN A POROUS MEDIUM
}

\author{
Olaleye Olalekan A. \\ Department of Statistics, \\ The Federal Polytechnic, Ede, \\ Osun State, Nigeria.
}

\author{
Olalude, Gbenga A. \\ Department of Statistics, \\ The Federal Polytechnic, Ede, \\ Osun State, Nigeria.
}

\author{
Amola, Benjamin A. \\ Department of Statistics, \\ The Federal Polytechnic, Ede, \\ Osun State, Nigeria.
}

\begin{abstract}
The effects of variable temperature on an unsteady Magnetohydrodynamics (MHD) free convective flow in a porous medium were investigated. The dimensional governing equations were reduced to nondimensional form with the use of some dimensionless quantities, resulting to nonlinear coupled partial differential equations. These equations were then solved using regular perturbation method. The physical parameters that arose in the analysis were examined on the Temperature and Velocity gradients, as well as the skin friction, which were later shown on graphs. Both velocity and skin friction decreased with increase in magnetic parameter but fluctuate with different values of frequency of oscillation. The temperature also decreased with increasing prandtl number. These outcomes however follow the results of existing literatures.
\end{abstract}

Keywords- Convective flow, Magnetohydrodynamics (MHD), magnetic parameter, perturbation method, skin friction, Prandtl number.

\section{INTRODUCTION}

The study of heat and mass flow has become very essential because of its wide range of applications in various fields of science, engineering and technology. Moreover, the MHD flows attract good attention and significant in several biological and engineering systems especially when the flow is considered over a permeable boundary layer.

In their study of effects of chemical reaction and radiation on unsteady MHD free convective fluid flow embedded in a porous medium with time-dependent suction with temperature gradient heat source, Seshaiah et al (2012) discovered that increase in the radiation parameter and Schmidt number both decreased the velocity of the fluid, while the magnetic field parameter also decreased the velocity for fixed permeability of the porous medium. Moreover, the magnetic parameter has remarkable effect on velocity profile but slight impact on temperature of the fluid (Noran et al, 2017).

Furthermore, it is confirmed by Kandasamy and Mohamad (2015) that temperature distribution in a nanofluid in the presence of thermal radiation with magnetic field significantly depends on the surface convection parameter. Mahender and Srikanth (2015) studied an unsteady MHD free convection and mass transfer flow past a porous vertical plate in presence of viscous dissipation. Kho et al (2017) investigated thermal radiation effect on MHD flow and heat transfer analysis of Williamson nanofluid past over a stretching sheet with constant wall temperature. Kumaresan et al (2017) worked on chemically reacting on MHD boundary layer flow of $\mathrm{CuOwater}$ and $\mathrm{MgO}$-water nanofluids past a stretching sheet in porous media with radiation absorption and heat generation/absorption. Nainaru and Satya (2017) studied unsteady MHD nanofluid flow over a stretching sheet with chemical reaction.

Moreover, the work of Harish, Sudheer and Satya (2017) on MHD mass transfer flow of an Eyring-Powell fluid over a stretching sheet revealed that the fluid velocity increased for the non-Newtonian fluid as compared with the corresponding Newtonian fluid for the effect of the induced magnetic field parameter.

This work thus focuses on effects of variable temperature on MHD free convective flow in a porous medium.

\section{MATHEMATICAL FORMULATION}

An unsteady two dimensional free convective MHD laminar flow of viscous incompressible and heat absorbing fluid pass a porous medium. All other fluid properties are assumed to be constant. The flow is infinite along vertical axis $\left(x^{\prime}-\right.$ axis $)$ in the direction which is opposite to the gravity and $y^{\prime}$-axis is taken to be normal to it. Since the flow is of extreme size along the vertical axis, the flow field becomes the function of $y^{\prime}$ and $t^{\prime}$ alone (Mohammed, 2013). The temperature at the surface of the porous plate was initially at equilibrium with the fluid, that is at $t^{\prime}=0$, but it was slowly raised with time, that is, at $t^{\prime}>0$. Under the above assumptions, the governing equations with the usual Boussinesq's approximation became.

Continuity equation 


\section{International Journal of Engineering Applied Sciences and Technology, 2019 \\ Vol. 4, Issue 7, ISSN No. 2455-2143, Pages 360-364 \\ Published Online November 2019 in IJEAST (http://www.ijeast.com)}

$$
\frac{\partial v^{\prime}}{\partial y^{\prime}}=0
$$

Momentum equation

$$
\frac{\partial u^{\prime}}{\partial t^{\prime}}=\vartheta \frac{\partial^{2} u^{\prime}}{\partial y^{\prime 2}}+g \beta\left(T^{\prime}-T_{\infty}^{\prime}\right)-\frac{\sigma B_{0}^{2} u^{\prime}}{\rho}
$$

Energy equation

$$
\rho C_{p} \frac{\partial T^{\prime}}{\partial t^{\prime}}=k \frac{\partial^{2} T^{\prime}}{\partial y^{\prime 2}}
$$

where $x^{\prime}$ and $y^{\prime}$ are dimensional distances along and perpendicular to the porous plate respectively. $t^{\prime}$ is the dimensional time. $u^{\prime}$ and $v^{\prime}$ are the components of dimensional velocities along $x^{\prime}$ and $y^{\prime}$ respectively. $\vartheta$ is the kinematic viscosity, $g$ is the acceleration due to gravity, $\beta$ the volumetric coefficient of thermal expansion, $T^{\prime}$ is the dimensional temperature, $\rho$ is the density, $k$ the thermal conductivity, $C_{p}$ is the specific heat capacity. The second term at the right hand side of the momentum equation represents the thermal buoyancy effect, while the third term stands for the induced magnetic field.

These are then subject to the following dimensional boundary conditions:

$$
\begin{aligned}
& u^{\prime}=U_{0}, \quad T^{\prime}=T_{w}^{\prime}+\lambda^{\prime} \cos \omega^{\prime} t^{\prime} \text { on } y^{\prime}=0 \\
& u^{\prime} \rightarrow U_{\infty}, \quad T^{\prime} \rightarrow T_{\infty}^{\prime} \quad \text { as } y^{\prime} \rightarrow \infty
\end{aligned}
$$

where $U_{0}$ is the initial or wall velocity, $T_{w}^{\prime}$ and $T_{\infty}^{\prime}$ are the wall and free stream temperatures respectively. $\omega^{\prime}$ and $\lambda^{\prime}$ are also the frequency and amplitude of the oscillations respectively.

Introducing the following dimensionless parameters as used by Akinpelu et al (2016), Akinpelu et al (2017), Mohammed (2013), Seshaiah et al (2013) and Fayza (2012):

$$
\begin{array}{lll}
u=\frac{u^{\prime}}{U_{0}}, & y=\frac{U_{0} y^{\prime}}{v}, & t=\frac{U_{0}^{2} t^{\prime}}{v} \\
\theta=\frac{T^{\prime}-T_{\infty}^{\prime}}{T_{w}^{\prime}-T_{\infty}^{\prime}}, & G r=\frac{g \beta \vartheta\left(T_{w}-T_{\infty}\right)}{U_{0}^{3}}, & P_{r}=\frac{\rho C_{p} v}{k}, \\
A_{0}=\frac{\lambda^{\prime}}{T_{w}^{\prime}-T_{\infty}^{\prime}}, & M=\frac{\sigma B_{0}^{2} v}{\rho U_{0}^{2}}
\end{array}
$$

The equations (2) and (3) reduced to:

$$
\begin{aligned}
& \frac{\partial u}{\partial t}=\frac{\partial^{2} u}{\partial y^{2}}+G r-u M \\
& \frac{\partial \theta}{\partial t}=\frac{1}{P_{r}} \frac{\partial^{2} \theta}{\partial y^{2}}
\end{aligned}
$$

Subject to the boundary conditions:

$$
\begin{array}{llll}
u=1, & \theta=1+A_{0} \cos \omega t & \text { on } & y=0 \\
u \rightarrow 0, & \theta \rightarrow 0 & \text { as } & y \rightarrow \infty
\end{array}
$$

\section{METHOD OF SOLUTION}

Equations (6) and (7) are coupled non-linear partial differential equations. In order to reduce these equations into ordinary differential equations and solved analytically, the regular perturbation method is employed with assumed solutions for the velocity and temperature gradient respectively is given as:

$$
\begin{aligned}
& u(y, t)=u_{0}(y)+\varepsilon e^{i \omega t} u_{1}(y)+o(\varepsilon)^{2} \\
& \theta(y, t)=\theta_{0}(y)+\varepsilon e^{i \omega t} \theta_{1}(y)+o(\varepsilon)^{2}
\end{aligned}
$$

Substituting equations (9) and (10) into (6) and (7) respectively, equating the harmonic and non-harmonic terms, and neglecting the higher order terms $o(\varepsilon)^{2}$ with further simplifications, these become

$$
\begin{aligned}
& u_{0}^{\prime \prime}-u_{0} M=-G r \theta \\
& u_{1}^{\prime \prime}-(M+i \omega) u_{1}=0 \\
& P_{r}^{-1} \theta_{0}^{\prime \prime}=0 \\
& \theta_{1}^{\prime \prime}-i \omega P_{r} \theta_{1}=0
\end{aligned}
$$

where the primes represent ordinary differentiation with respect to $y$.

Subject to the following corresponding boundary conditions: $u_{0}=1, u_{1}=0, \theta_{0}=1+A_{0} \cos \omega t, \theta_{1}=0$ on $y=0$ $u_{0} \rightarrow 0, u_{1} \rightarrow 0, \theta_{0} \rightarrow 0, \theta_{1} \rightarrow 0 \quad$ as $\quad y \rightarrow \infty$

Solving equations (11) - (14) subject to the boundary conditions (15), the resulted velocity and temperature distributions are:

$$
\begin{aligned}
& u=\cosh (\sqrt{M} y)-\sinh (\sqrt{M} y)-\frac{G r}{M}\left[1+A_{0} \cos \omega t\right]+\varepsilon e^{i \omega t-(\sqrt{M+i \omega}) y} \\
& \theta=1+A_{0} \cos \omega t+\varepsilon e^{i \omega t-\left(\sqrt{i \omega P_{r}}\right) y}
\end{aligned}
$$

The skin friction which is an important physical parameter for boundary flow of this type is given as:

$$
\begin{aligned}
& \tau=\left.\left(\frac{d u}{d y}\right)\right|_{y=0}= \\
& -\sqrt{M} \sinh (\sqrt{M} y)-\sqrt{M} \cosh (\sqrt{M} y)-(\sqrt{M+i \omega}) \varepsilon e^{i \omega t-(\sqrt{M+i \omega}) y}
\end{aligned}
$$




\section{International Journal of Engineering Applied Sciences and Technology, 2019 \\ Vol. 4, Issue 7, ISSN No. 2455-2143, Pages 360-364 \\ Published Online November 2019 in IJEAST (http://www.ijeast.com)}

\section{RESULTS AND DISCUSSION}

For the purpose of proper discussion, the numerical results of the transient velocity, temperature and skin friction were computed and displayed on graphs. The physical quantities that arose from these, namely: the magnetic parameter $(\mathrm{M})$, Prandtl number $\left(P_{r}\right)$ and frequency of oscillation $(\omega)$ were examined on the Temperature and Velocity gradients, as well as the skin friction.

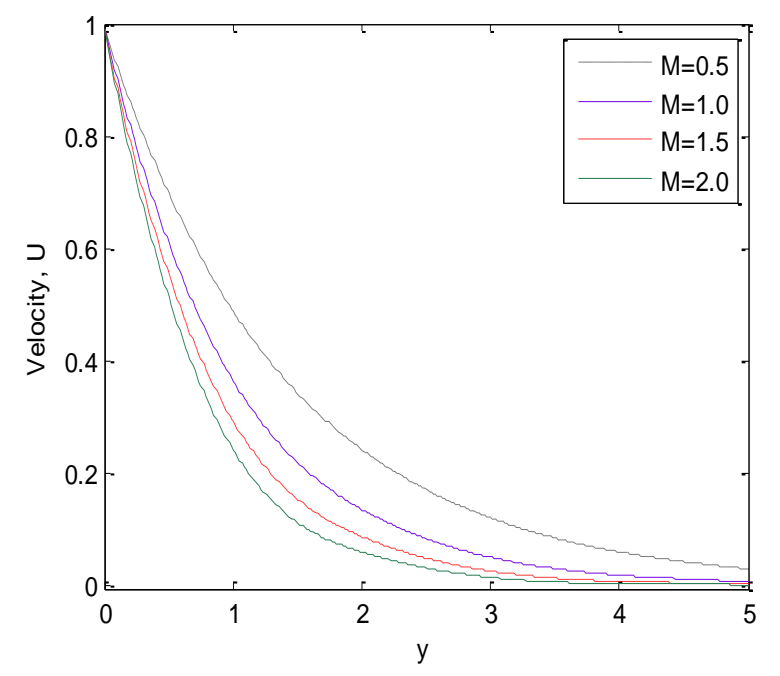

Figure 1: Velocity profile against spanwise coordinate y for different values of magnetic field $(\mathrm{M})$ with

$P_{r}=0.71, A_{0}=1.0, \omega=\pi / 2, \varepsilon=0.01, t=10, G r=2.0$

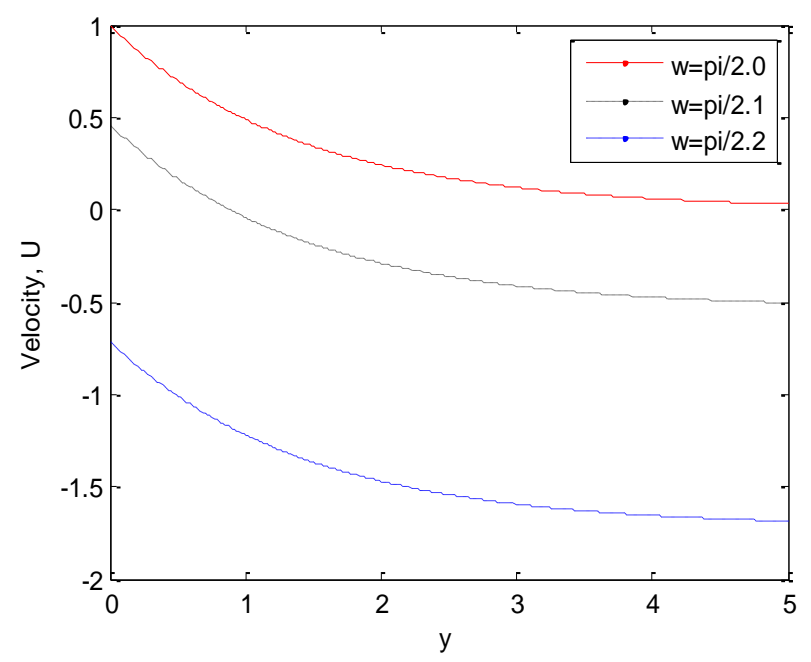

Figure 2: Velocity profile against spanwise coordinate y for different values of frequency of oscillation $(\omega)$ with $P_{r}=0.71, A_{0}=1.0, M=0.5, \varepsilon=0.01, t=10, G r=2.0$

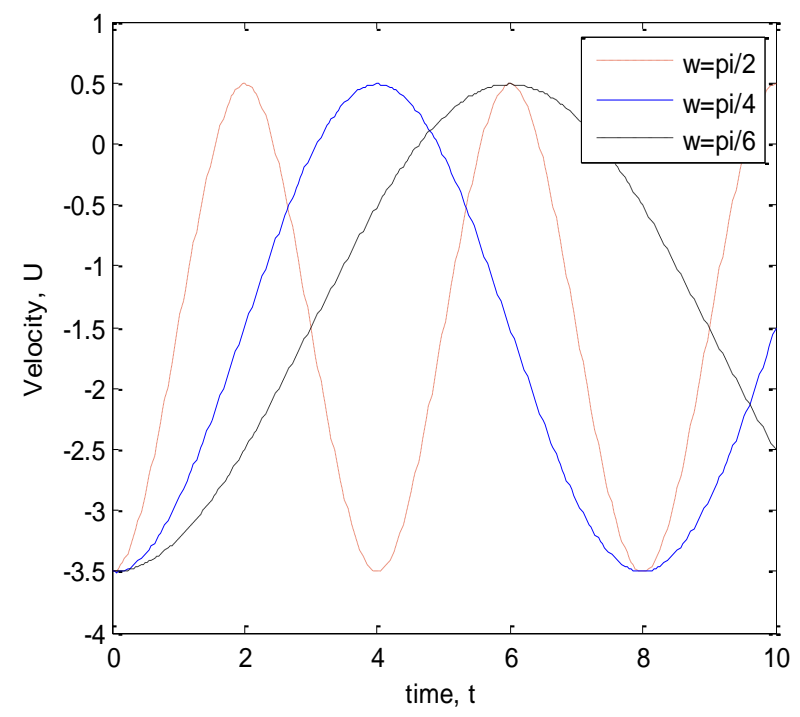

Figure 3: Velocity profile against time for different values of frequency of oscillation $(\omega)$ with

$P_{r}=0.71, A_{0}=1.0, M=0.5, \varepsilon=0.01, y=1.0, G r=2.0$

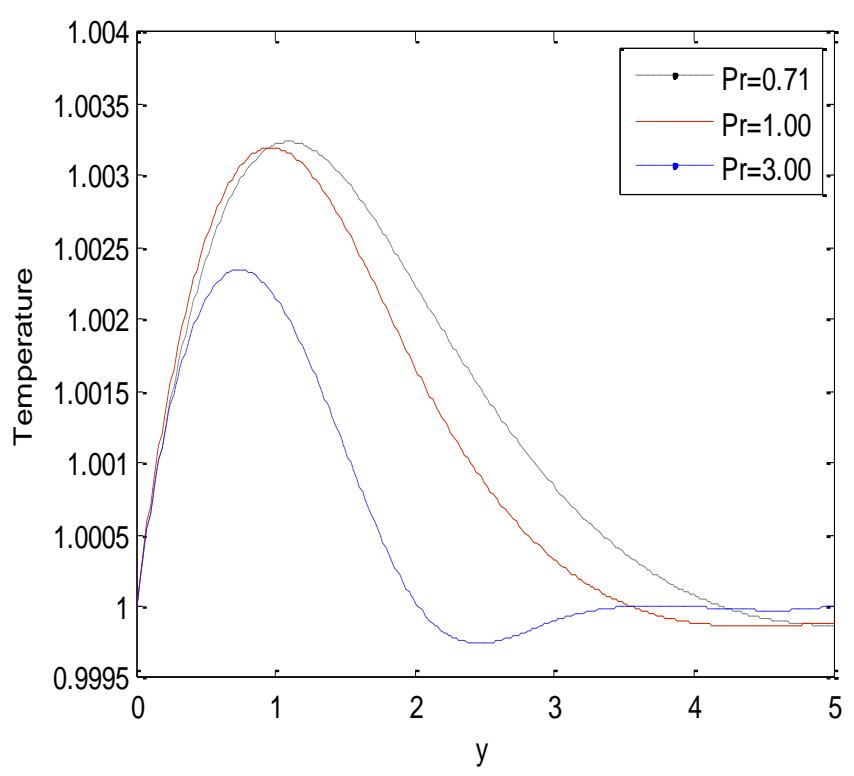

Figure 4: Temperature profile against spanwise coordinate y for different values of Prandtl number $\left(P_{r}\right)$ with

$$
A_{0}=1.0, \omega=\pi / 2, \varepsilon=0.01, t=1.0
$$


International Journal of Engineering Applied Sciences and Technology, 2019

Vol. 4, Issue 7, ISSN No. 2455-2143, Pages 360-364

Published Online November 2019 in IJEAST (http://www.ijeast.com)

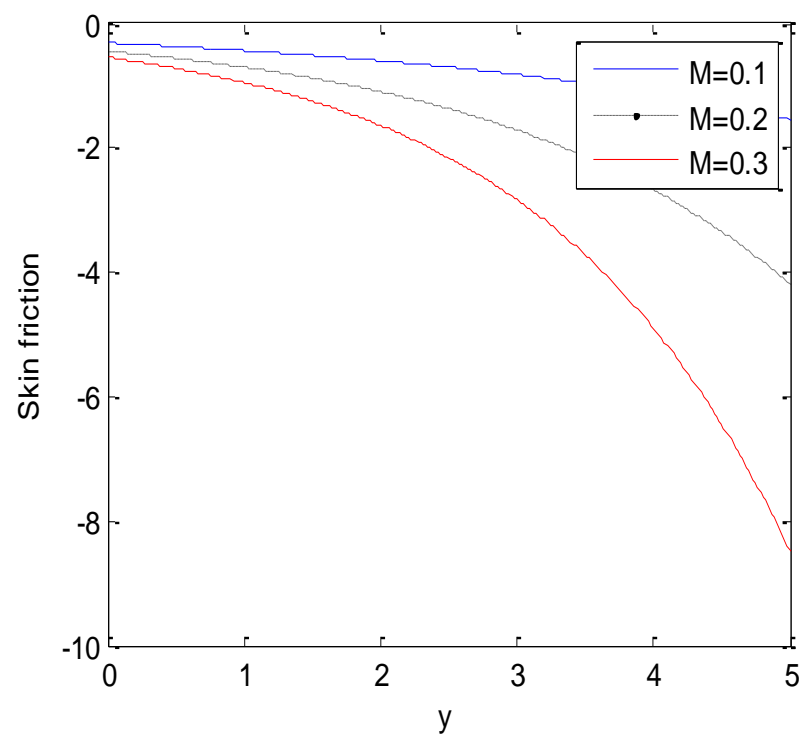

Figure 5: Skin friction against spanwise coordinate y for different values of magnetic field $(\mathrm{M})$ with

$$
P_{r}=0.71, \omega=\pi / 2, \varepsilon=0.01, t=10
$$

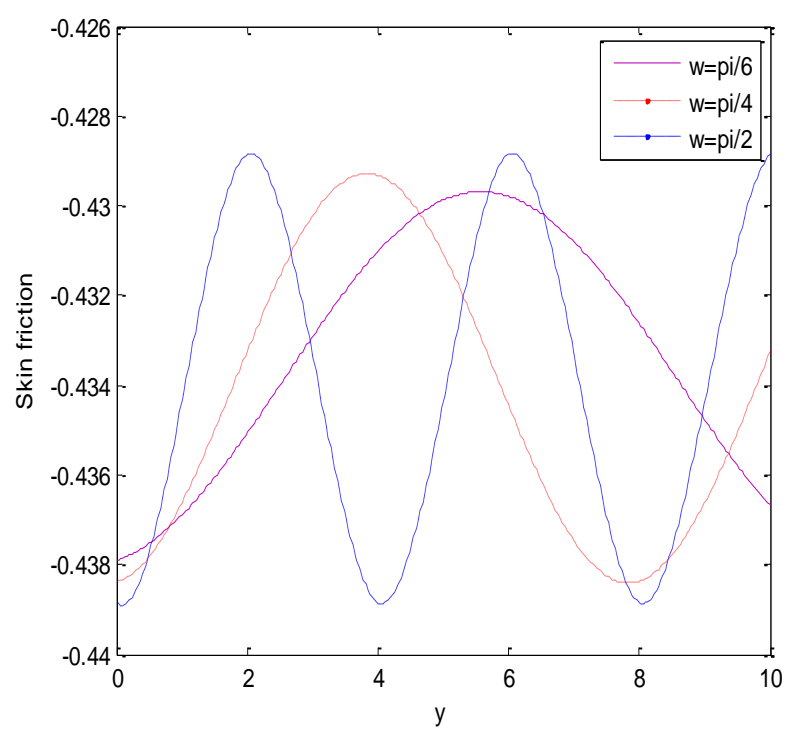

Figure 6: Skin friction against spanwise coordinate y for different values of frequency of oscillation $(\omega)$ with

$$
P_{r}=0.71, M=0.1, \varepsilon=0.01, t=10
$$

Figure 1 which is the velocity profile against spanwise coordinate $\mathrm{y}$ for different values of magnetic field (M) represents the effects of the induced magnetic field parameter on the velocity field. The result shows that increase in the induced magnetic field decreased the velocity of the fluid. This corresponds to the result of Seshaiah et al (2013) among host of others.
Figure 2 shows the velocity profile against spanwise coordinate $\mathrm{y}$ for different values of frequency of oscillation $(\omega)$. The results revealed that as the frequency of oscillation is decreasing, the velocity is also decreasing.

However, figure 3 presents the sinusoidal variation of the velocity with time at different values of frequency of oscillation. The decreasing frequency of oscillation increased the period of oscillation, while the amplitude remains constant according to the boundary condition.

Figure 4 is the temperature profile against spanwise coordinate $\mathrm{y}$ for different values of Prandtl number. The increasing Prandtl number decreases the temperature, which is in line with existing works like that of Mohammed (2013). This behaviour however changed as the distance approached around 3.5. This is because of the peculiarity of Prandtl number associated with different fluid of interest.

Figure 5 is the skin friction against spanwise coordinate y for different values of magnetic field. The result follows that of the velocity above. The increase in the induced magnetic field leads to decrease in the skin friction.

Moreover, figure 6 shows the sinusoidal variation of the skin friction at different values of frequency of oscillation. The amplitude is no more fixed, since $A_{0}$ was not in the skin friction solution obtained in the last section

\section{CONCLUSION}

The effects of the variable temperature on an unsteady two dimensional free convective MHD laminar flow of viscous incompressible and heat absorbing fluid pass a porous medium have been studied and the following conclusions were made. The induced magnetic field parameter decreased both the velocity and the skin friction. There is sinusoidal variation in the velocity and skin friction with time and distance respectively at different values of frequency of oscillation. Moreover, the increase in prandtl number decreased the temperature till a distance of about 3.5.

\section{REFERENCE}

1. Akinpelu F. O., Alabison R. M. and Olaleye O. A. (2016). "Variations in Ground Temperature in the presence of Radiative Heat Flux and Spatial Dependent soil thermophysical property". International Journal of Statistics and Applied mathematics 2016, 2(1): 57-63.

2. Akinpelu F.O., Olaleye O. A. and Adewoye S. K. (2017). "The effects of some physical parameters on ground temperature with time-dependent suction velocity in the presence of internal heat generation". Imperial Journal of Interdisciplinary Research (IJIR). Vol-3, Issue-8, 2017 ISSN: 2454-1362, http://www.onlinejournal.in

3. Fayza Mohammed Nasser El-Fayer (2012). Effects of Chemocal reaction on the unsteady free convection 
Flow past an Infinte Vertical Permeable Moving Plate with Variable Temperature. Journal of Surface Engineered Materials and Advanced Technology, 2:100-109

4. Harish Babu D., Sudheer Babu M. and Satya Narayana P. V. (2017). MHD mass transfer flow of an EyringPowell fluid over a stretching sheet. IOP Conf. Series: Materials Science and Engineering 263 (2017) 062013 doi:10.1088/1757-899X/263/6/062013

5. Kandasamy R. and Mohamad R. (2015). Radiative Heat Transfer on Nanofluids Over a Porous Convective Surface in the Presence of Magnetic Field. Journal of Applied Mechanical Engineering. 4(4):172. doi:10.4172/2168-9873.1000172

6. Kho Y B, Hussanan A., Mohamed M K A, Sarif N M, Ismail Z and Salleh M Z (2017). Thermal radiation effect on MHD Flow and heat transfer analysis of Williamson nanofluid past over a stretching sheet with constant wall temperature. Journal of Physics: Conf. Series $\mathbf{8 9 0}$ (2017) 012034 doi $: 10.1088 / 1742-$ 6596/890/1/012034

7. Kumaresan E., Vijaya Kumar A. G. and Rushi Kumar B. (2017). Chemically reacting on MHD boundary layer flow of CuOwater and $\mathrm{MgO}$-water nanofluids past a stretching sheet in porous media with radiation absorption and heat generation/absorption. Conf. Series: Materials Science and Engineering 263 (2017) 062017 doi:10.1088/1757-899X/263/6/062017

8. Mahender D. and Srikanth Rao P. (2015). Unsteady MHD free convection and mass transfer flow past a porous vertical plate in presence of viscous dissipation. Journal of Physics: Conference Series 662012012 doi:10.1088/1742-6596/662/1/012012

9. Mohammed Ibrahim S. (2013). "Radiation Effects on Mass Transfer Flow through a Highly Porous Medium with Heat Generation and Chemical Reaction". IRSN Computational Mathematics, Volume 2013, Article ID 765408.

10. Nainaru Tarakaramu and Satya Narayan P.V. (2017). Unsteady MHD nanofluid flow over a stretching sheet with chemical reaction. IOP Conf. Series: Materials Science and Engineering 263062030 doi:10.1088/1757-899X/263/6/062030

11. Noran Nur Wahida Khalili, Abdul Aziz Samson, Ahmad Sukri Abdul Aziz and Zaileha Md Ali (2017). Journal of Physics. Conf. Series 890 (2017) 012025 doi :10.1088/1742-6596/890/1/012025

12. Seshaiah B., Varma S.V.K. and Raju M. C. (2013). The effects of Chemical Reaction and Radiation on Unsteady MHD Free Convective Fluid Flow Embeded in a Porous Medium with Time-Dependent Suction with Temperature Gradient Heat Source. International Journal of Scientific Knowledge. Computing and Information Technology. Vol. 3, No. 2. ISSN 23051493 